\title{
Zukunftspraktiken in Organisationen
}

\section{Kommentar zum Beitrag von Koch/Krämer/Reckwitz/Wenzel „Zum Umgang mit Zukunft in Organisationen - eine praxistheoretische Perspektive“"}

\author{
Jens Beckert
}

Online publiziert: 21. September 2016

(C) Der/die Autor(en) 2016. Dieser Artikel ist eine Open-Access-Publikation.

Zusammenfassung Der Kommentar diskutiert den Artikel ,Zum Umgang mit Zukunft in Organisationen - eine praxistheoretische Perspektive“ von Koch, Krämer, Reckwitz und Wenzel.

Schlüsselwörter Zukunft in Organisationen · Performativität · Politik der Erwartungen $\cdot$ Steuerungstheorie $\cdot$ Praxistheorie

In den Sozialwissenschaften lässt sich derzeit ein neues Interesse an den temporalen Strukturen sozialer Prozesse erkennen. Dabei gerät insbesondere die Zukunft in den Blick. Nicht im Sinne einer Futurologie, wie sie in den Sozialwissenschaften der 1960er und 1970er Jahre starken Einfluss hatte, sondern aus handlungstheoretischer Perspektive: Wenn zukünftige Entwicklung offen, kontingent und somit ungewiss ist, wie gelangen intentional rationale Akteure zu Entscheidungen, deren Folgen für sie bedeutsam aber nicht vorhersehbar sind?

Traditionell sind Erklärungen in den Sozialwissenschaften darauf gerichtet, gegenwärtiges Handeln aus in der Vergangenheit geformten Strukturen zu erklären etwa Institutionen, Netzwerken, sozialstrukturellen Attributen oder kognitiven Skripten. Auf die Rolle der Zukunft ausgerichtete Erklärungsmodelle drehen die Kausalität hingegen um: Es sind die mit den Entscheidungen verbundenen Erwartungen zukünftiger Handlungsresultate, die das gegenwärtige Handeln erklären. Nicht nur „history matters“, „the future matters“!

Die Wirtschaftswissenschaften und auch die Managementlehre sind die sozialwissenschaftlichen Fächer, in denen auf die Zukunft rekurrierende Erklärungen schon seit langem selbstverständlicher Teil normativer Theorien sind: Grundlage moderner

J. Beckert (两)

Max-Planck-Institut für Gesellschaftsforschung (MPIfG), Paulstr. 3, 50676 Köln, Deutschland

E-Mail: beckert@mpifg.de 
finanztheoretischer Modelle der Investitionsrechnung ist, dass rationale Entscheidungen auf der Diskontierung von in der Zukunft erwarteten Zahlungsströmen basieren. Spieltheoretische Modelle erklären gegenwärtiges Handeln aus dem ,Schatten der Zukunft.“ Die Managementlehre entwickelt Planungstechniken, die gegenwärtiges Handeln anleiten sollen, indem zukünftige Konsequenzen der Entscheidungen antizipiert und Handlungswege über viele Stufen präzise beschrieben werden.

Diese Modelle der ökonomischen Theorie und der Managementlehre gehen zumeist von der rationalen Antizipierbarkeit der Zukunft aus, zumindest in einem probabilistischen Sinn. Angesichts der Offenheit der Zukunft und zumeist unvollständiger Informationsausstattung der Akteure ist dies jedoch eine unhaltbare Annahme. Diese Einsicht ist auch in Teilen der Wirtschaftswissenschaften und der Betriebswirtschaftslehre angekommen. Doch die daraus entstehende Frage erweist sich als ungeheuer schwer zu beantworten: Wie werden Entscheidungen von intentional rationalen Akteuren getroffen, wenn Handlungsergebnisse im Sinne von Frank Knights Unterscheidung nicht einfach riskant sind, sondern ungewiss?

Der hier kommentierte Artikel geht dieser Frage am Beispiel des Managementprozesses nach, untersucht sie also für organisationales Handeln. Dies ist weiterführend, da Organisationshandeln in der aktuellen sozialwissenschaftlichen Literatur zur Zukunftsorientierung bisher eher wenig Aufmerksamkeit erlangt hat.

Die Autoren schlagen eine praxistheoretische Orientierung vor, was ich hier nicht weiter kommentiere, da ich diese Perspektive weitgehend teile. Knapp zusammengefasst geht es darum, die tatsächlichen Praktiken der Akteure zu beobachten und zum Ausgangspunkt der Theoriebildung zum Umgang des Managements mit der ungewissen Zukunft zu machen. In dem Artikel changieren die Autoren allerdings immer zwischen zwei Perspektiven, die beide relevant sind, jedoch voneinander zu trennen wären. Zum einen geht es ihnen um die „Analyse der Formen der Zukunftsbearbeitung, durch die Organisationen das Kommende hervorbringen und verarbeiten“ (S. 3). Zum anderen geht es ihnen darum, selbst ,eine praxistheoretische Perspektive der Zukunftsbearbeitung“ (S. 5) zu entwickeln, also „um die Weiterentwicklung steuerungstheoretischer Ansätze“ (S. 1). Im Sinne von Niklas Luhmann gehen hier Beobachtungen zweiter Ordnung mit Beobachtungen erster Ordnung durcheinander und der Artikel hätte deutlich an Klarheit gewonnen, wenn sich die Autoren allein auf die Analyse des Zukunftsumgangs im Management konzentriert hätten.

Ich gehe in meinem Kommentar auf vier Themenfelder ein, die über die von den Autoren gemachten Ausführungen weiterer Entwicklung und Präzisierung bedürften. Drei dieser Themenfelder werden von den Autoren zumindest angerissen, eines fehlt. Die vier Punkte sind Performativität, Macht, Pluralität und Steuerungstheorie. Zum Abschluss werde ich noch einige Ideen zu dem in dem Artikel angeregten organisationstheoretischen Forschungsprogramm darlegen.

Performativität Der Begriff der Performativität wird von den Autoren an verschiedenen Stellen des Textes angeführt. Seit Einführung dieses Begriffs in der Wirtschaftssoziologie durch Callon (1998) und MacKenzie (2006) hat sich Performativität zu einem modischen Term mit häufig unpräziser Verwendung entwickelt. Alle Effekte sozialen Handelns werden mit dem Attribut performativ versehen. Doch es kommt darauf an, hier begrifflich präzise zu sein. Für die Autoren des Artikels 
meint Performativität schlicht, dass die ,zukünftige Gegenwart“ von gegenwärtigen Entscheidungen abhängig ist. Hierfür bräuchte man den Begriff der Performativität nicht, viel einfacher ließe sich von der Realitätswirkung von Zukunftsentwürfen sprechen. Denn Performativität im Sinne von Callon und MacKenzie meint etwas sehr viel Spezifischeres: ökonomische Modelle führen durch ihre Anwendung die Realität immer näher an die Modelle selbst heran. Diese Wirkung ökonomischer Modelle konnte anhand einiger gut ausgesuchter Fälle empirisch gezeigt werden. Doch lässt sich gerade in der Untersuchung der Formen der Zukunftsbearbeitung in Organisationen darlegen, dass der Performativitätsthese im Sinne von Callon und MacKenzie gegenüber Vorsicht angeraten ist. Denn wenn man von der Kontingenz der Zukunft ausgeht, dann ist es wenig wahrscheinlich, dass eine in der Gegenwart behauptete Zukunft auch tatsächlich zur ,zukünftigen Gegenwart“ wird. Und tatsächlich lassen sich sofort unzählige Beispiele dafür anführen, dass theoriegenerierte Erwartungen oder organisationale Planungen nicht die tatsächliche spätere Entwicklung beschreiben und somit sich die Realität der Theorie gerade nicht anpasst. Das gilt für die Finanzkrise 2007 ebenso wie für das Scheitern von Unternehmenszusammenschlüssen oder organisationalen Restrukturierungsprozessen. Vorstellungen über Zukunft haben (möglicherweise) Wirkungen, doch die Unbestimmtheit dieser Wirkungen muss gerade ein wichtiger Bestandteil der Theorie bleiben.

Macht Es ist überraschend, in einem Artikel über den Umgang mit Zukunft in Organisationen nicht einmal den Begriff Macht zu lesen. Wenn es in Organisationen um die Bildung von Erwartungen zukünftiger Entwicklungen geht, dann geht es nicht vornehmlich um empirische Vielfältigkeit oder die Koordination von Wissen, sondern es geht um Machtausübung. Dies gilt sowohl innerhalb der Organisation als auch im Hinblick auf das wettbewerbliche Umfeld. Zukunftsprojektionen sind in Organisationen umkämpft, es gibt eine „Politik der Erwartungen“ (Beckert 2016). Denn bei Zukunftsprojektionen geht es immer auch um die Verteilung von Ressourcen für Projekte; es geht um Entscheidungen, die distributive Konsequenzen für die Organisationsmitglieder haben. Wird das Projekt dieser oder jener Abteilung vom Vorstand genehmigt? Doch die mit Zukunftsprojektionen verbundenen „fiktionalen Erwartungen“ (Beckert 2016) lassen sich auch als zentrales Element des Marktkampfes (Max Weber) verstehen. Investoren davon zu überzeugen, dass die eigene gerade in der Entwicklung befindliche Technologie dem technologischen Ansatz des Konkurrenten überlegen ist, sichert für das Unternehmen die finanziellen Ressourcen, mit denen überhaupt erst herausgefunden werden kann, ob die Entwicklung technisch und wirtschaftlich tragfähig ist. Der amerikanische Organisationswissenschaftler James March hat dies eindrucksvoll formuliert: „,Sooth-sayers create sheltered worlds of ignorance, ideology and faith. Within the shell that they provide, craziness is protected long enough to elaborate its challenge to orthodoxy“" (1995, S. 437). Zukunftsprojektionen als Teil der Mikropolitik in Organisationen und als zentrales Moment von Wettbewerb zwischen Unternehmen zu verstehen, ist unabdingbar, gerade auch aus einer praxistheoretischen Perspektive.

Pluralität Eine interessante Beobachtung der Autoren besteht in ihrer Feststellung, dass sich in Unternehmen gleichzeitig vielfältige Vorstellungen über Zukünfte 
und planerisches Vorgehen finden. Obwohl dem Management eigentlich klar ist, dass rationale Planungsverfahren angesichts komplexer Umwelten notwendigerweise unzuverlässig sein müssen, werden solche Praktiken weiter verfolgt. Daneben jedoch etablieren sich neue Techniken, die von der Notwendigkeit permanenter Revision von Erwartungen im Lichte neuer unvorhersehbarer Entwicklungen ausgehen. Dieses Phänomen der „Zukunft im Plural“ innerhalb von Unternehmen wird von den Autoren jedoch nicht weiter untersucht. Warum bestehen in Organisationen gleichzeitig verschiedene Zukunftskonzepte und Techniken der Planung? Ist eine solche Heterogenität angesichts von Ungewissheit selbst ein Verfahren, durch das Organisationen Robustheit erlangen (Bronk und Jacoby 2016; Stark 2009)? Wird durch diese Pluralität Macht verteilt? Wird dadurch Kreativität befördert? Handelt es sich schlicht um ein Übergangsphänomen? Dienen einige der Verfahren der Legitimation, andere der faktischen Entscheidungsfindung? Eine Vielzahl an interessanten Fragen ließe sich an die von den Autoren gemachte Beobachtung anschließen.

Steuerungstheorie Ein weiterer interessanter, in dem Artikel jedoch ebenfalls nicht systematisch ausgeführter Gedanke bezieht sich auf die Verwendung des Begriffs Steuerungstheorie. Die Steuerungstheorie hat ihre Anfänge im Planungsoptimismus der 1950er und 1960er-Jahre und ist weit über die Managementforschung hinaus bedeutsam gewesen, insbesondere für die politikwissenschaftliche PolicyForschung (Mayntz 2004). Die Autoren beziehen den Begriff vor allem auf Planungstheorien der frühen Managementforschung (S. 10) und argumentieren überzeugend, dass solche Ansätze angesichts überwältigender Kontingenz (Ungewissheit!) wenig realitätstauglich sind. Die Managementforschung hat sich darauf mit neuen, die Kontingenz von Entscheidungen betonenden Ansätzen eingestellt. Die bei den Autoren zwar im Hintergrund mitzuschwingende, aber nicht explizit gemachte Frage lautet, inwiefern die beobachteten auf Erwartungsbildung setzenden Managementpraktiken selbst die zentrale neue Steuerungstechnik ökonomischer (und politischer?) Prozesse sein könnte. Beispiele, die diese Überlegung stützen, lassen sich leicht finden. Die seit den 1980er-Jahren von den westlichen Zentralbanken verfolgte Politik der ,forward guidance“ setzt auf die möglichst zielgenaue Steuerung von Inflationserwartungen der Marktteilnehmer, deren Handlungen dann so ausfallen, dass die Geldwertstabilität erhalten bleibt. Aber auch in der Technologieentwicklung finden sich Beispiele für erwartungsbasierte Handlungssteuerung. Ein besonders prominentes Beispiel ist Moore's Law, also die in der Halbleiterindustrie seit den 1960er Jahren verfestigte Erwartung der Verdoppelung der Prozessorengeschwindigkeit alle zwei Jahre. Zukunftsprojektionen als Steuerungsinstrument zu untersuchen bietet eine fruchtbare Forschungsperspektive, die an eine zentrale sozialwissenschaftliche Begrifflichkeit anschließt und zu Erkenntnissen führen könnte, die weit über die Erforschung von Managementprozessen hinausgeht.

Forschungsprogramm Die Autoren möchten mit ihrem Artikel ein organisationstheoretisches Forschungsprogramm (S. 32) entwickeln, das seinen Ausgangspunkt bei den Zukunftserwartungen von Unternehmen nimmt. Dem möchte ich voll und ganz zustimmen. Tatsächlich könnte ein solches Forschungsprogramm außerordentlich fruchtbar sein. Doch die Autoren bleiben hinsichtlich der Ausformulierung ei- 
nes solchen Programms merkwürdig abstrakt. In dem Artikel erfährt der Leser mehr über (bekannte?) Konzepte der Praxistheorie als über die Praxis der Unternehmen. Lediglich in Halbsätzen wird angedeutet, womit sich das anvisierte Forschungsprogramm empirisch beschäftigen könnte: so wird von ,visionären Zukunftserzählungen“ (S. 15) berichtet und später von Leitbildern in Organisationen, Ansprachen bei Firmenjubiläen und den Projektionen von Forschungsabteilungen (S. 27). In der Tat sind dies Beispiele für mögliche interessante Forschungsprojekte, anhand derer sich der praktische Umgang von Unternehmen mit einer unsicheren Zukunft untersuchen ließe. Doch dies sollte viel genauer und auch systematischer ausbuchstabiert werden. Sofort fallen einem viele andere mögliche Forschungsthemen ein: die Arbeit von Planungsabteilungen (sei es für die Unternehmensstrategie oder die Produktentwicklung) und auch die Untersuchung der in Unternehmen angewandten Techniken zur Unterstützung von komplexen Entscheidungen. Die Rolle von makroökonomischen Prognosen, von Szenarien und Risikoanalysen. Dabei wird sich finden, dass viele dieser Techniken eine völlig unrealistische Sicherheit bei der Prognose zukünftiger Entwicklungen zur Schau stellen, doch auch hieran lassen sich wichtige organisationssoziologische Fragestellungen anschließen: Welche Funktionen erfüllen scheinbar präzise Vorhersagen im Entscheidungsprozess und in den Mikroprozessen der Organisation? Schließlich könnte es um die genaue Untersuchung der Mechanismen gehen, mit denen bestimmte Zukunftsvisionen glaubhaft werden. Welche diskursiven und narrativen Techniken lassen sich hierbei erkennen? Welche Rolle spielt die Positionierung der Akteure im Feld? Wie werden Narrative zerstört?

Die Untersuchung der Perzeption der Zukunft in Organisationen bietet eine außerordentlich fruchtbare Forschungsperspektive. Die Autoren des Artikels liegen richtig in ihrem Ansatz, hierfür insbesondere die Praktiken der Akteure zu untersuchen. Die Hoffnung wäre, aus empirischen Fallstudien generelles Wissen zum Verständnis von Handeln unter Bedingungen von Ungewissheit zu erlangen und die Steuerungstheorie so zu erneuern, dass Erwartungen und Projektionen der Zukunft darin die Rolle spielen, die sie in der Unternehmenspraxis längst haben.

Open access funding provided by Max Planck Society.

Open Access Dieser Artikel wird unter der Creative Commons Namensnennung 4.0 International Lizenz (http://creativecommons.org/licenses/by/4.0/deed.de) veröffentlicht, welche die Nutzung, Vervielfältigung, Bearbeitung, Verbreitung und Wiedergabe in jeglichem Medium und Format erlaubt, sofern Sie den/die ursprünglichen Autor(en) und die Quelle ordnungsgemäß nennen, einen Link zur Creative Commons Lizenz beifügen und angeben, ob Änderungen vorgenommen wurden.

\section{Literatur}

Beckert J (2016) Imagined futures. Fictional expectations and capitalist dynamics. Harvard University Press, Cambridge

Bronk R, Jacoby W (2016) Uncertainty and the dangers of monocultures in regulation, analysis, and practice. MPIfG Discussion Paper 16/6. Max-Planck-Institut für Gesellschaftsforschung, Köln

Callon M (Hrsg) (1998) The laws of the markets. Blackwell, Oxford

MacKenzie D (2006) An engine, not a camera: how financial models shape markets. MIT Press, Cambridge 
March JG (1995) The future, disposable organizations and the rigidities of imagination. Organization 2:427-440

Mayntz R (2004) Governance Theory als fortentwickelte Steuerungstheorie? MPIfG Working Paper 04/1. Max-Planck-Institut für Gesellschaftsforschung, Köln

Stark D (2009) The sense of dissonance: accounts of worth in economic life. Princeton University Press, Princeton 